\title{
A relação entre 0 mapeamento de processos e a modelização no contexto da gestão do conhecimento: estudo de caso aplicado em uma biblioteca digital
}

\author{
Raíssa Yuri Hamanaka* \\ Filipi Miranda Soares*
}

Artículo recibido:

27 de julio de 2018

Artículo aceptado:

13 de marzo de 2019

Artículo de investigación
Resumo

Busca-se demonstrar as relações entre o mapeamento de processos com a modelização conceitual dentro do contexto da Gestão do Conhecimento (GC) e como atividade a ser realizada pelos profissionais da informação. O mapeamento é a primeira atividade de modelagem de processos e antecede a modelagem conceitual, que está inserida no contexto da GC, que visa identificar e compartilhar os conhecimentos tácitos dos indivíduos, de forma a tornar processos mais eficazes e facilitar a tomada de decisões estratégicas. $\mathrm{O}$ artigo aborda uma revisão de literatura sobre a GC, a modelização e modelagem conceitual, e as relaciona com o

* Universidade Federal de Minas Gerais (UFMG), Brasil raissa0201@gmail.com filipims@ufmg.br INVESTIGACIÓN BIBLIOTECOLÓGICA, vol. 33, núm. 81, octubre/diciembre, 2019, México,
ISSN: 2448-8321 pp. 223-240 
mapeamento de processos. Em seguida, o mapeamento é exemplificado por meio do estudo de caso no sistema de registro de teses e dissertações do Instituto Superior de Contabilidade e Administração do Porto.

Palavras-chave: Mapeamento de Processos; Gestão do Conhecimento; Modelização Conceitual; Modelagem Conceitual

La relación entre el mapeo de procesos y la modelización en el contexto de la gestión del conocimiento: estudio de caso aplicado en una biblioteca digital Raíssa Yuri Hamanaka y Filipi Miranda Soares

\section{RESUMEN}

Se intenta demostrar las relaciones entre el mapeo de procesos con la modelización conceptual dentro del contexto de la gestión del conocimiento (GC) y como actividad a ser realizada por los profesionales de la información. El mapeo es la primera actividad del modelado de procesos y precede al modelado conceptual, que está insertado en el contexto de la GC, y cuyo fin es identificar y compartir los conocimientos tácitos de los individuos para hacer procesos más eficaces y facilitar la toma de decisiones estratégicas. El artículo aborda una revisión de literatura sobre la GC, la modelización y el modelado conceptual, y los relaciona con el mapeo de procesos. En seguida, el mapeo es ejemplificado por medio del estudio de caso en el Sistema de Registro de Tesis y Disertaciones del Instituto Superior de Contabilidad y Administración de Porto.

Palabras clave: Mapeo de Procesos; Gestión del Conocimiento; Modelización Conceptual; Modelado Conceptual

The relationship between process mapping and conceptual modeling on knowledge management: a case study applied in a digital library

Raissa Yuri Hamanaka and Filipi Miranda Soares

\section{Abstract}

This work aims to demonstrate the relationship between process mapping and conceptual modeling 
within the context of Knowledge Management (KM) and as an activity to be carried out by information professionals. Mapping is the first activity of process modeling and precedes conceptual modeling that is embedded in the context of KM, which aims to identify and share the tacit knowledge of individuals with the intention of making processes more efficient and facilitate strategic decision making. The article addresses a literature review on KM and conceptual modeling, and relates them to process mapping. Next, the mapping is exemplified by means of a case study in the registration system of theses and dissertations of Porto's Accounting and Business School.

Keywords: Process Mapping; Knowledge Management; KM; Conceptual Modeling

\section{INTRODUÇÃO}

$A_{\text {objetivo primordial das pesquisas em Ciência da Informação (CI), que }}^{\text {comunicac̃a efetiva do conhecimento registrado é provavelmente o }}$ envolve diferentes disciplinas. No âmbito das organizações, por exemplo, há a necessidade de se administrar a produção do conhecimento da staff e dos processos organizacionais, pois, de acordo com Choo (2001), esse conhecimento possui alto valor para o desenvolvimento estratégico. Tal disciplina é denominada Gestão do Conhecimento (GC) e é exatamente sobre este campo que este trabalho se desenvolve, mais especificamente na etapa denominada mapeamento.

O objetivo do artigo é relacionar o mapeamento de processos com a CI, sob o ponto de vista da representação do conhecimento, mais especificamente da modelização conceitual, levando-se em consideração que antes de se representar um domínio é preciso mapeá-lo e definir que tipos de informações, documentos, processos, etc. devem ser representados. Mas também, sob o ponto de vista da GC, tendo em vista que antes de tornar um conhecimento tácito em explícito é necessário mapeá-lo e definir em quais setores de uma determinada organização os conhecimentos são utilizados ou produzidos. Como objetivo específico, propõe-se elaborar um modelo para o mapeamento de processos.

Ao longo do texto, será apresentada uma revisão de literatura sobre a GC nas organizações, sobre a modelização conceitual e sobre o mapeamento de 
processos, discutindo-se sobre o mapeamento de processos e suas relações com a GC e com a modelização conceitual e, um estudo de caso que apresenta o mapeamento de processos do sistema de registro de teses e dissertações do Instituto Superior de Contabilidade e Administração do Porto (ISCAP), desenvolvido por Soares e Machado (2017), que exemplifica o passo-a-passo para a execução de um mapeamento de processos.

\section{Gestão Do Conhecimento}

O desenvolvimento de todo trabalho implica em aprendizagem. Segundo Davenport e Prusak $(1998,52)$ "toda organização saudável gera e usa conhecimento". Mesmo os trabalhos mais mecânicos e repetitivos exigem que os indivíduos que os desempenham memorizem processos e se familiarizem com certas práticas de forma a alcançar resultados mais eficientes em menor tempo. Nesse processo, o indivíduo está produzindo conhecimento para aprimorar suas atividades. Pessoas podem ocupar por décadas um mesmo cargo em uma organização, o que as torna extremamente experientes no campo em que atuam. Um profissional experiente em sua função traz benefícios para a organização, mas o que fazer caso este decida deixar o cargo ou se aposentar? Esse é um dos problemas que, segundo Choo (2006), podem ser resolvidos com a aplicação de práticas de GC.

Segundo Davenport e Prusak (1998: 52), "À medida que as organizações interagem com seus ambientes, elas absorvem informação, a transformam em conhecimento e tomam decisões baseadas na combinação de suas experiências, valores e regras internas". Essa produção de conhecimento acontece em três etapas. Vários autores ilustraram o processo de gestão do conhecimento em três etapas, a saber:

a) Nonaka e Takeuchi (1995), com o modelo espiral do conhecimen to:

- from tacit to tacit;

- from explicit to explicit;

- from tacit to explicit.

b) Davenport e Prusak (1998):

- knowledge generation;

- knowledge codification and coordination;

- knowledge transfer.

c) Choo $(2001 ; 2006)$, com o modelo de organização do conhecimento:

- sense making;

- knowledge creating;

- decision making. 
Os autores apresentam diferentes modelos, divididos em etapas, para representar o processo de GC nas organizações. Apesar de apresentarem propostas estruturadas de formas diferentes, os autores concordam com uma questão fundamental: é preciso transformar o conhecimento tácito em explícito para que as organizações possam utilizar todo o potencial deste insumo.

De maneira geral, os modelos de organização do conhecimento propõem três etapas: a identificação do conhecimento tácito ou know how das pessoas ao executarem suas atividades diárias; a criação do conhecimento explícito por meio do registro conhecimento tácito das pessoas e a ação tomada a partir do novo conhecimento (seja a tomada de decisão ou o compartilhamento das informações). O mapeamento de processos se daria segunda etapa do modelo de organização do conhecimento, através da descrição detalhada do passo-a-passo das ações executadas pelos agentes.

Segundo Rautenberg, Steil e Todesco (2011: 31) dentre os instrumentos de GC, estão: Compartilhamento de Melhores Práticas, Comunidades de Prática, Mapas de Conhecimento, Lições Aprendidas, Tutoria, E-learning, Gestão de Conteúdo ou Páginas Amarelas.

De acordo com Nonaka (2007), o conhecimento tácito é pessoal e subjetivo, o que o torna difícil de ser formalmente expresso para que outros indivíduos possam captá-lo e reutilizá-lo no contexto organizacional.

Do ponto de vista organizacional, o conhecimento humano ou capital intelectual é o ativo mais valioso da empresa, e o objetivo da Gestão do Conhecimento é identificá-lo, registrá-lo e compartilhá-lo (Hommerding e Vergueiro, 2004). Caracteriza-se como o "[...] O conjunto de competências de um indivíduo", obtidas a partir de "[...] sua formação acadêmica, cursos de especialização, idiomas, relacionamento pessoais e cognitivos [...]” dentre outros. Se gerenciado de forma efetiva, pode gerar informações estratégicas para a tomada de decisão na organização. Todavia, mapear o conhecimento tácito não é uma tarefa simples, uma vez que os conhecimentos de um indivíduo estão intrinsecamente relacionados às suas experiências de vida e às influências dos ambientes onde vive (Hommerding e Vergueiro, 2004: 34).

Conforme Hommerding e Vergueiro (2004), o profissional da informação está fortemente relacionado com a gerência do mapeamento de processos e com a GC, pois é o mais capacitado na organização e estruturação de informações organizacionais que serão transformadas em conhecimento.

O mapeamento de processos se torna uma ferramenta para a conversão do conhecimento tácito para explícito e podem ser aplicadas diferentes metodologias para localizar os fluxos informacionais de uma organização e mapeá-los. 
Logo, infere-se que o mapeamento de informações ou do conhecimento é processo chave na GC. Moresi, Ramos e Prado (2010: 108) estabelecem a relação entre o mapeamento de processos e a GC:

A construção de um mapa de informações permite facilitar a localização e o acesso às informações armazenadas nos diversos sistemas corporativos. O seu uso subsidiará os gerentes da área de tecnologia da informação e gestores de negócio da organização estudada, no atendimento às suas necessidades de informações no que diz respeito a localizá-las na empresa e identificar a quais processos de negócio estão associadas.

Entretanto, o mapeamento é apenas uma das etapas da modelagem de sistemas de informação, que gera modelos relacionais dos processos da organização como um todo. Será discutido o passo seguinte na modelagem de processos.

\section{Mapeamento De PROCESSOS}

Antes de se definir o mapeamento de processos é importante localizá-lo dentro da modelagem de processos, que segundo Villela (2000) citado por Miranda (2010: 101) "[...] busca verificar o alinhamento entre as atividades executadas e o objetivo do negócio (eficácia), e a adequação entre o consumo de recursos e os produtos gerados (eficiência)". Ao analisar a modelagem de processos com uma visão holística, é possível identificar quatro fases, de acordo com Miranda (2010: 102):

planejamento (diagnóstico ou definição dos processos a serem mapeados e da metodologia a ser utilizada); o mapeamento dos processos; o monitoramento (criação dos mecanismos de controle e verificação de qualidade); e uma fase de intervenções para atingir mais eficiência e efetividade na gestão dos processos de informação [...].

O mapeamento de processos é a primeira etapa da modelagem de processos. Consiste em analisar e recolher informações dos focos que produzem e/ ou usam informação dentro da organização e dos seus fluxos. Uma organização é composta por pessoas que executam tarefas e que se organizam em departamentos. Estas tarefas são denominadas processos e o mapeamento desses permite "que a instituição analise os fluxos de trabalho partindo dos processos mais abrangentes em direção aos menos abrangentes", o que consiste em uma visão top down do fluxo de processos, que facilita identificar 
"os macroprocessos, os subprocessos e as atividades envolvidas em todo o fluxo" (De Paula e Valls, 2014: 141).

O objetivo do mapeamento de processos vai de encontro ao objetivo da Arquitetura da Informação, isto é, estruturar informações para "[...] tornar acessível o que já existe na organização” (Moresi, Ramos e Prado, 2010: 103).

Existem várias técnicas ou metodologias para mapear processos, cada uma com símbolos próprios, assim como com vantagens e desvantagens. Antes de se definir a metodologia de mapeamento é necessário se considerar uma série de variáveis, tais como: o objetivo que se deseja alcançar, o contexto, o tempo, os resultados esperados, a partir daí justifica-se o uso de determinada metodologia em detrimento de outras (Miranda, 2010: 104). Algumas das técnicas mais comuns são: o BPM (Business Process Modelling); a ARIS (Architecture of Integrated Information Systems); o formulário SIPOC (Supply, Inputs, Process, Output e Consumers); o Servpro (junção das palavras "serviço" e "processo"); o roteiro do Instituto Nacional de Pesquisas Espaciais (INPE) (De Paula e Valls, 2014: 144-145); o fluxograma ou diagrama de fluxo; o IDEF (Integration Definition for Function Modelling); o LOVEM-E (Enhanced Line of Visibility Enterprise Modelling) e o E-TOM (Enbanced Telecommunications Operations Map) (Miranda, 2010: 104).

Não é objetivo deste artigo detalhar todas as metodologias de mapeamento. Todavia, como o BPM foi utilizado no estudo de caso apresentado a seguir optou-se por descrevê-lo:

BPM é uma abordagem funcional que trata do planejamento, modelagem, controle e execução de processos de trabalho, orientando as atividades. A notação [Business Process Modelling Notation] BPMN foi desenvolvida pelo Business Process Management Iniciative (BPMI.org) e permite representar as atividades de um processo, sem esquecer os fluxos informacionais relacionados a essas atividades, as pessoas que as executam e seus papéis, a sequência lógica na qual são executadas as tarefas e os eventos ligados a elas. (Miranda, 2010: 108)

Apesar de existirem diversas metodologias para mapeamento de processos, na literatura da CI há uma carência da descrição de como são feitos esses mapas, conforme apontam Moresi, Ramos e Prado (2010: 104) “[...] foi feito um apanhado geral dos trabalhos realizados e relevantes com o tema desta pesquisa. A partir deste apanhado verificou-se que não existiam procedimentos relatados a respeito de como fazer um mapeamento de informações".

Os autores sugerem um conjunto de elementos básicos que um mapa de informações deve conter: 
[...] o que (what) - que corresponde às informações; como (how) - quais os processos de negócio a informação está associada; onde (where) - local onde as informações podem ser encontradas; e quem $(w h o)$ - que corresponde a qual área da organização é responsável pelo sistema de informação. (Moresi, Ramos e Prado, 2010: 105-107)

Ao final da revisão de literatura sobre mapeamento de processos surge uma questão: por que mapear? O mapeamento permite a visualização descritiva e detalhada dos processos de uma organização, através dele é possível identificar as falhas, problemas e restrições nas atividades realizadas, isto é, os pontos fracos e fortes, as ameaças e oportunidades das mesmas com o objetivo de reduzir tempo e custo.

\section{ModelizaçÃo E MODELAgem CONCEITUAL}

Segundo Silva, Farinelli e Almeida (2014), modelar é um processo mental de abstrair conceitos que representem a realidade, portanto, a modelagem conceitual é a representação de uma realidade (sob o ponto de vista de quem a representa) através da definição dos conceitos e de seus relacionamentos.

Nesse contexto, o mapeamento de informações sobre processos seria uma atividade facilitadora da descrição das abstrações da realidade. É uma forma de representar o conhecimento tácito em um modelo formal que posteriormente pode ser automatizado.

De acordo com Campos (2004a), percebe-se a diferença entre modelar um domínio do conhecimento com base em modelos pré-existentes (Teoria da Classificação Facetada, Teoria do Conceito, Teoria da Terminologia, Orientação a Objetos, Ontologia Formal) e modelizar um domínio com base em princípios de teorias representacionais. Ao extrair os principais conceitos de teorias representacionais, os modelizadores teriam capacidade metodológica para representar um contexto informacional, seus objetos e relacionamentos, com base em princípios norteadores (métodos de raciocínio, escolha de definição dos objetos a serem representados, definição de como se relacionam e modelo de representação gráfica) (Campos, 2004a; 2004b).

Ainda conforme Campos (2004a: 102) "Esta perspectiva restaura a liberdade do modelizador porque quanto mais ele teoriza os seus métodos mais possibilidade ele tem de não ficar refém de um dado modelo específico". Modelar é conhecer e a modelização é a construção do conhecimento a partir da representação de um domínio observado (Campos, 2004a: 102). 
A partir da definição de Campos (2004b) sobre modelização de domínios do conhecimento é possível fazer uma distinção entre modelagem conceitual e modelização conceitual. A primeira é composta por modelos de representação da informação que formalizam conceitos e seus relacionamentos, enquanto a modelização envolve princípios que regem a representação de um domínio do conhecimento específico. Conforme a mesma autora (2004b: 23), os princípios que norteiam a modelização são:

O primeiro princípio diz respeito ao método de raciocínio utilizado para a organização do conhecimento dentro de um domínio. O segundo analisa como está definido o objeto de representação, ou seja, qual é a unidade de conhecimento que se vai representar. $\mathrm{O}$ terceiro diz respeito à relação entre os objetos, objetivando verificar as possibilidades de ligação/separação semânticas entre os conceitos de um dado domínio. O quarto evidencia as formas de representação gráfica que um modelo pode adotar.

Segundo esses princípios de modelização é necessário se definir o objeto ou unidade de informação a ser representada. Nesta etapa o mapeamento das informações é necessário para a definição dos requisitos informacionais do domínio a ser representado. Por meio dele é possível mapear os fluxos informacionais do contexto estudado, os processos realizados por agentes, as atividades meio e fim realizadas durante os mesmos e os produtos gerados pelas atividades (como a criação de documentos).

\section{ESTUDO DE CASO}

Esta seção apresenta o mapeamento de processos realizado por Soares e Machado (2017) no sistema de registro de teses e dissertações do ISCAP como parte da disciplina de Modelação de Sistemas de Informação, componente curricular da Licenciatura em Ciências e Tecnologias da Documentação e Informação (LCTDI).

\section{Metodologia para o mapeamento de processos}

De forma geral, a metodologia utilizada para mapear os processos do sistema de registro de teses e dissertações do ISCAP foi executada conforme o modelo apresentado na Figura 1. 


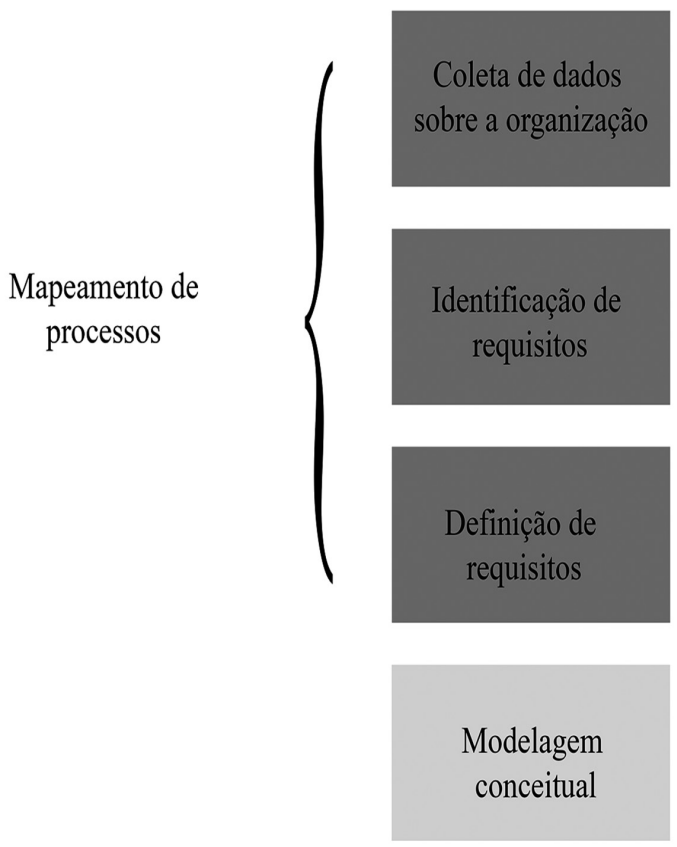

Figura 1. Mapeamento de processos Fonte: desenvolvido pelos autores, 2018

A primeira etapa do mapeamento consiste em levantar dados sobre a organização, o que pode ser feito aplicando-se diferentes técnicas, como entrevistas, questionários, análise de documentos e outros. Essas técnicas podem ser combinadas para se obterem melhores resultados. Pode-se, por exemplo, analisar documentos produzidos como resultado de um processo executado dentro da organização e realizar entrevistas com as pessoas envolvidas naquele processo de forma a melhorar o entendimento sobre o mesmo.

$\mathrm{Na}$ etapa de coleta de dados é comum que se obtenha informações que não serão relevantes para a modelagem do sistema. $\mathrm{Na}$ segunda etapa do mapeamento essas informações devem ser eliminadas. $\mathrm{Na}$ identificação dos requisitos devem-se analisar as informações obtidas a partir das transcrições de áudios de entrevistas, respostas de questionários, análise de documentos e quaisquer outras fontes de informação e identificar o que são os requisitos dentre as informações coletadas.

Os requisitos, segundo Ribas (2007: 64), "são fundamentalmente uma descrição de suas funções e restrições, buscando permitir a compreensão do problema a ser solucionado pelo sistema". 
A identificação de requisitos pode ser feita em texto corrido, de forma objetiva. Identificados os requisitos, é preciso defini-los. A definição de requisitos de um sistema de informação consiste em classificá-los em duas categorias: requisitos funcionais e não funcionais. Segundo Sommerville (2011: 59):

Requisitos funcionais: são declarações de serviços que o sistema deve fornecer, de como o sistema deve reagir a entradas específicas e de como o sistema deve se comportar em determinadas situações. Requisitos não funcionais: são restrições aos serviços ou funções oferecidos pelo sistema. Incluem restrições de timing, restrições no processo de desenvolvimento e restrições impostas pelas normas.

Os requisitos não funcionais podem ser classificados, segundo Rodrigues (2017), em tecnológico, desempenho, usabilidade, disponibilidade e segurança. O Quadro 1 apresenta a definição desses requisitos.

\begin{tabular}{|l|l|}
\hline \multicolumn{1}{|c|}{$\begin{array}{c}\text { Requisito não } \\
\text { funcional }\end{array}$} & \multicolumn{1}{c|}{ Definição } \\
\hline Tecnológico & $\begin{array}{l}\text { Descrevem características operacionais relacionadas com o ambiente tecnoló- } \\
\text { gico (hardware e software) da organização }\end{array}$ \\
\hline Desempenho & $\begin{array}{l}\text { Descrevem características operacionais relacionadas com o desempenho } \\
\text { do sistema de informação, tais como, o tempo de resposta, ou o tempo de } \\
\text { introdução de dados. }\end{array}$ \\
\hline Usabilidade & $\begin{array}{l}\text { Descrevem características operacionais relacionadas com os utilizadores, } \\
\text { processamentos incorretos, tratamentos de erros e recuperação de dados. }\end{array}$ \\
\hline Disponibilidade & $\begin{array}{l}\text { Descrevem a dependência do sistema, tais como são tratadas as situações } \\
\text { de indisponibilidade do sistema, sobrecarga de utilizadores, processamentos } \\
\text { incorretos, tratamento de erros e recuperação de dados. }\end{array}$ \\
\hline Segurança & $\begin{array}{l}\text { Descreve os acessos dos utilizadores a determinadas funcionalidades ou dados } \\
\text { e as condições sobre as quais o acesso é permitido. }\end{array}$ \\
\hline
\end{tabular}

Quadro 1. Requisitos não funcionais

Fonte: Rodrigues (2017)

Uma vez classificados os requisitos, segue-se para a etapa de modelagem conceitual, na qual serão criadas relações entre os requisitos definidos de forma a esquematizar a representação dos fluxos informacionais que o sistema deve suportar. Segundo Moresi, Ramos e Prado (2010: 108) para o sucesso da implantação do mapeamento de processos recomenda-se:

[...] divulgar a importância da arquitetura da informação e do mapa de informações para a organização, além de seus benefícios; atribuir a função de mapeamento a um profissional da área de informação, que deverá estar dedicado 
exclusivamente à execução das atividades do processo de mapeamento; definir uma diretriz no processo de desenvolvimento de sistemas da organização para quando houver modificação das necessidades de informações de um sistema, que esta seja comunicada ao arquiteto da informação para atualização do mapa de informações existente; oficializar a atividade de validação da categorização das informações pelo arquivista da organização; usar como referência um Código de Classificação de Documentos para a classificação de todas as informações da organização; e divulgar constantemente, para toda a organização, o mapa de informações construído.

Portanto, para que o mapeamento seja bem-sucedido é necessário combinar profissionais capacitados (arquitetos da informação), e deixar claro para os indivíduos que atuam na organização que o mapeamento trará benefícios, logo espera-se que todos contribuam com o arquiteto da informação.

\section{Aplicaçáo da metodologia de mapeamento de processos na Biblioteca Digital do ISCAP e resultados}

O primeiro passo foi realizar um diagnóstico da organização. É importante iniciar o diagnóstico coletando dados sobre a estrutura da organização e representá-los como um organograma, pois a partir dele será possível tomar conhecimento sobre "onde os processos operam, as estruturas formais de comunicação existentes no ambiente, as técnicas de gestão e as tecnologias utilizadas na organização" (Miranda, 2010: 102). O organograma do ISCAP pode ser genericamente representado conforme a Figura 2.

A biblioteca do ISCAP é responsável por registrar as teses e dissertações no Repositório Científico do Politécnico do Porto. Após entrevista com a bibliotecária responsável pelo setor e análise de documentos institucionais, foram selecionadas informações relevantes e mapeadas conforme o Quadro 2

0 estudante entrega cópias da tese à divisão acadêmica em número suficiente para que cada membro do júri receba uma cópia da tese (uma cópia em papel e um arquivo digital em um CD). A divisão acadêmica encaminha as teses ao júri.

0 júri determina se 0 trabalho foi aprovado ou não. Caso seja aprovado, 0 júri envia à divisão acadêmica a ata de defesa assinada pelos membros da banca; sugere as alterações a serem feitas e 0 aluno terá um prazo de 30 (trinta) dias para entregar a versão final da tese à divisão acadêmica. Ao receber a tese, a seção acadêmica faz o registro do documento no Software RENATES. A instituição tem um prazo de 60 dias (sessenta dias) para efetuar o registro do documento no RENATES. 
A divisão acadêmica envia a tese impressa em papel, $0 \mathrm{CD}$ e um formulário (contendo algumas informações sobre a tese, como nome do autor, título, orientador) à biblioteca. $\mathrm{A}$ biblioteca confere $0 \mathrm{CD}$; se 0 arquivo contido no $\mathrm{CD}$ respeitar às regras da biblioteca, 0 arquivo será carregado no Repositório Científico do Instituto Politécnico do Porto (RECIPP). Ao ser carregado no RECIPP, o documento é automaticamente carregado no Repositórios Científicos de Acesso Aberto de Portugal (RCAPP) e recebe um identificador, o qual deve ser enviado à divisão acadêmica - a biblioteca gera um formulário e 0 envia a divisão acadêmica. Se 0 arquivo do CD não respeitar às normas institucionais, será enviado novamente à divisão acadêmica que deverá contatar 0 aluno e exigir que entregue $0 \mathrm{CD}$ com 0 arquivo correto.

A cópia da tese em papel será catalogada e disponibilizada no acervo da biblioteca.

0 aluno deve assinar um termo de responsabilidade autorizando ou não a disponibilização online do documento. Se sim, esse será registrado no RECIPP; se não, esse será guardado no arquivo da biblioteca e não ficará disponível ao público.

Quadro 2. Descritivo de informações do sistema de registro de teses e dissertações do ISCAP Fonte: Soares e Machado (2017: 4-5)

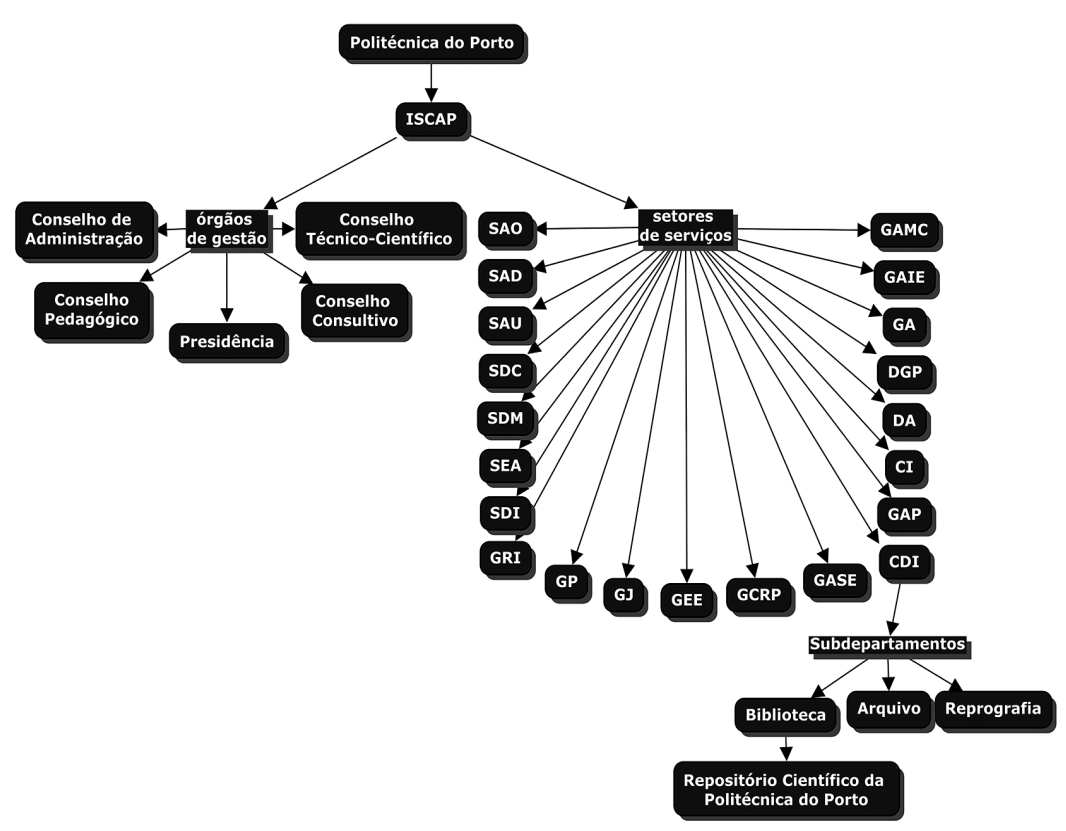

Figura 2. Organograma do ISCAP

Fonte: elaborado pelos autores a partir de dados do ISCAP, 2018.

https://www.iscap.ipp.pt/iscap/organica/servicos

Legenda: CDI) Centro de Documentação e Informação; CI) Centro de Informática; DA) Divisão Académica; DGP) Divisão de Gestão de Pessoas; GA) Gabinete Alumni; GAIE) Gabinete de Apoio à Inovação em Educação; GAMC) Gabinete de Planeamento, Avaliação e Melhoria Contínua; GAP) Gabinete de Apoio a Projetos; GASE) Gabinete de Ambiente e Simulação Empresarial; GCRP) Gabinete de Comunicação e Relações Públicas; GEE) Gabinete de Estágios E Empregabilidade; GJ) Gabinete Jurídico; GP) Gabinete do Património; GRI) Gabinete de Relações Internacionais; SAD) Secretariado de Apoio aos Docentes; SAO) Secretariado de Apoio aos Órgãos; SAU) Serviço Auxiliar; SDC) Serviço de Contabilidade; SDM) Serviço de Manutenção; SEA) Serviço de Expediente e Arquivo; SDT) Serviço de Tesouraria 
O texto que preenche o Quadro 2 é o primeiro mapa de processos, denominado diagnóstico por Miranda (2010). Posteriormente, foi necessário simplificar a descrição dos processos a nível de requisitos, o que gerou o seguinte conjunto:

a) receber tese; b) fazer registro RENATES; c) 60 dias para realizar registro; d) enviar tese à biblioteca; e) o arquivo deve respeitar às regras da biblioteca; f) carregar arquivo no RECIPP; g) documento é automaticamente carregado no RCAPP; h) gerar identificador; i) catalogar tese; j) assinar termo de responsabilidade; k) disponibilizar documento online (no RECIPP); 1) consultar teses. (Soares e Machado, 2017:5)

O passo seguinte, seguindo a ordem do modelo proposto na Figura 1, foi a definição dos requisitos do sistema. Os requisitos foram classificados em funcionais e não funcionais, de acordo com a categorização proposta por Sommerville (2011), e os não funcionais foram subclassificados como tecnológico, desempenho, usabilidade, disponibilidade e segurança, baseado na classificação proposta por Rodrigues (2017) apresentada no Quadro 1. Os resultados da definição dos requisitos estão apresentados no Quadro 3.

\begin{tabular}{|l|l|}
\hline \multicolumn{1}{|c|}{ Texto } & \multicolumn{1}{c|}{ Classificação do requisito } \\
\hline Receber tese & Requisito funcional \\
\hline Fazer registro RENATES & Requisito funcional \\
\hline 60 dias para realizar registro & Requisito não funcional: desempenho \\
\hline Enviar tese à biblioteca & Requisito funcional \\
\hline 0 arquivo deve respeitar às regras da biblioteca & Requisito não funcional: segurança \\
\hline Carregar arquivo no RECIPP & Requisito não funcional: tecnológico \\
\hline Documento é automaticamente carregado no RCAPP & Requisito não funcional: tecnológico \\
\hline Gerar identificador & Requisito funcional \\
\hline Catalogar tese & Requisito funcional \\
\hline Assinar termo de responsabilidade & Requisito não funcional: segurança \\
\hline Disponibilizar documento online (no RECIPP) & Requisito não funcional: tecnológico \\
\hline Consultar teses & Requisito funcional \\
\hline
\end{tabular}

Quadro 3. Definição de requisitos

Fonte: Soares e Machado (2017: 5)

A partir dos requisitos funcionais identificados no sistema de registro de teses e dissertações, foi gerado um modelo BPMN (Figura 3) por meio do software Signavio. A modelização conceitual dos requisitos levantados no 
mapeamento envolve análise mais profunda dos conceitos que cada tarefa representa para que seja possível criar as relações entre os processos do sistema de informação. É preciso entender bem o contexto organizacional e, se necessário, retornar ao primeiro texto do mapeamento (exemplificado no Quadro 2), onde os requisitos estão contextualizados e há explicação mais detalhada sobre cada processo.

É possível observar a partir do modelo as tarefas executadas em cada departamento e as relações entre elas. É possível, então, detectar problemas no fluxo das tarefas como, por exemplo, a biblioteca receber um CD com arquivo inválido ou corrompido. Esse problema acontece porque a divisão acadêmica não verifica o conteúdo do CD quando o recebe do aluno, que muitas vezes não salvou o arquivo da forma correta e detecta-se tal problema apenas quando o arquivo chega à biblioteca digital, o que dificulta a obtenção do arquivo correto, uma vez que com o diploma em mãos dificilmente o aluno retornará ao instituto para entregar o arquivo correto. A solução proposta para este problema é transferir a tarefa de verificação do arquivo digital do CD (denominada Analisa CD no modelo BPMN da Figura 3) para a Divisão Acadêmica.

A etapa seguinte à modelagem seria corrigir os erros detectados no modelo BPMN para otimizar os processos falhos e gerar um novo modelo. Para isso, seria necessário que os indivíduos responsáveis por desempenhar tais processos dentro da organização concordassem em mudar a forma como executam suas tarefas. Além disso, o modelo poderia ser automatizado para gerar um sistema de gerenciamento de informações.

Pode-se resumir a metodologia aplicada da seguinte maneira:

a) primeira etapa: diagnóstico da instituição, por meio de entrevista de análise de conteúdo documental;

b) segunda etapa: elaboração do descritivo do processo de registro de teses e dissertações com base no diagnóstico;

c) terceira etapa: identificar as principais atividades do descritivo e classificá-las em requisitos funcionais ou não funcionais;

d) quarta etapa: modelagem do processo de registro de teses e dissertações utilizando linguagem BPMN;

e) quinta etapa: identificar problemas do processo modelado;

f) sexta etapa: propor soluções aos problemas detectados;

g) sétima etapa: a partir das soluções apresentadas, gerar um novo modelo BPMN (a ser realizada). 


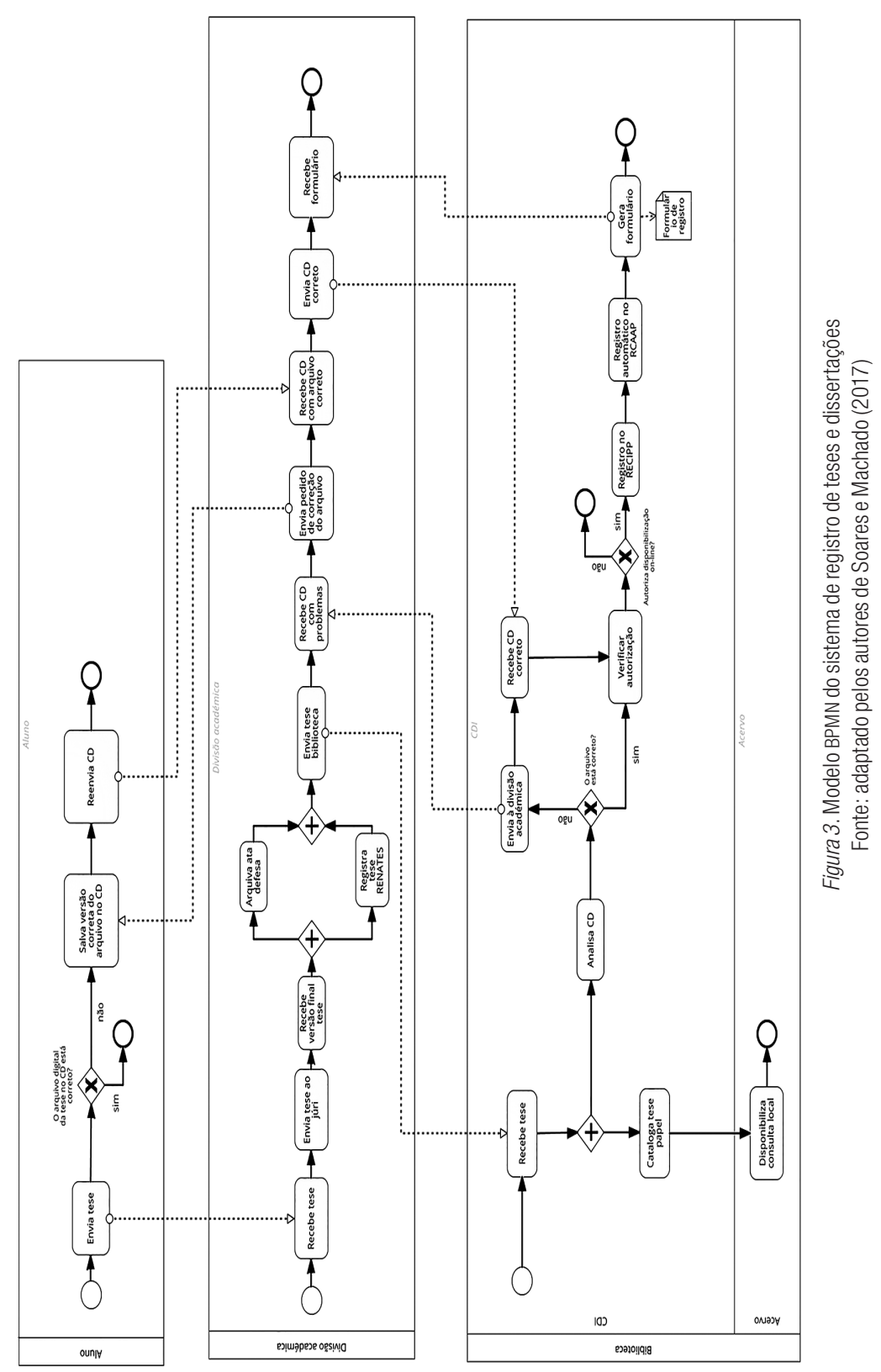




\section{Considerações Finais}

Tendo em vista o atual contexto informacional, em que há um grande volume de informações não estruturadas sendo produzidas indiscriminadamente nas organizações, encontrar a informação certa no momento certo se torna uma tarefa estratégica e financeiramente importante. O mapeamento de processos é uma atividade que visa apontar os fluxos informacionais de uma organização para proporcionar melhorias no desempenho de atividades. O sistema modelado permite recuperar as informações necessárias para execução de tarefas, o que inclui a tomada de decisões, de forma mais eficiente, ou seja, encontrar a informação relevante em tempo hábil. Este estudo se propôs a demonstrar a relação entre o mapeamento de processos e a modelagem ou modelização conceitual e os processos da Gestão do Conhecimento.

O mapeamento de processos na Biblioteca Digital do ISCAP permitiu identificar problemas nas atividades realizadas no processo de registro de teses e dissertações. A partir disso, foi apresentada uma solução potencial para o problema. Caso a solução fosse adotada, poderia aumentar a eficiência do processo descrito e evitar a perda de tempo e do acesso às teses e dissertações.

Portanto, o mapeamento de processos possibilita a descrição das atividades realizadas em uma organização e a detecção de erros na execução de tarefas. A partir do mapeamento, é possível propor soluções para aumentar a eficiência dos processos da organização.

\section{REFERÊNCIAS}

Campos, M. L. A. 2004a. “O ensino de representação de domínios de conhecimento: uma postura teórico-metodológica”, en (re)significação do processo de ensino/ aprendizagem em Biblioteconomia e Ciência da Informação: novas abordagens didático-pedagógicas, editado por M. E. F. Rodrigues y B. S. A. Campello, 101-118. Niterói: Intertexto; Rio de Janeiro: Interciência.

Campos, M. L. A. 2004b. "Modelização de domínios de conhecimento: uma investigação de princípios fundamentais”. Ciência da Informação 33 (1): 22-32.

Choo, C. W. 2001. "The knowing organization as learning organization". Education + Training 43 (4/5): 197-205.

Choo, C. W. 2006. The knowing organization: how organizations use information to construct meaning, create knowledge, and make decisions. Oxford: Oxford University Press.

Davenport, T. H. y L. Prusak. 1998. Working Knowledge: How Organizations Manage What They Know. Brighton: Harvard Business School Press.

De Paula, M. A. e V. M. Valls, 2014. "Mapeamento de processos em bibliotecas: revisão de literatura e apresentação de metodologias”. Revista Digital de Biblioteconomía e Ciência da Informação 12 (3): 136-156. 
Hommerding, N. M. S. y W. Vergueiro. 2004. "Profissionais da informação e o mapeamento do conhecimento nas organizações: o caso da KPMG Brasil”. Revista Digital de Biblioteconomia e Ciência da Informação 2 (1): 17-36.

Miranda, S. V. 2010. "A gestão da informação e a modelagem de processos". Revista do Serviço Público 61 (1): 97-112.

Moresi, E. A. D., R. G. C. Ramos y H. A. Prado, 2010. "Mapeamento de informações organizacionais: um estudo na Embrapa”. TransInformação 22 (2): 101-110.

Nonaka, I. 2007. "The Knowledge-Creating Company”. Harvard Business Review. http:// www.citizenartist.eu/wp-content/uploads/2017/02/PrelimReading4The-Knowledge-Creating-Company.pdf.

Nonaka, I. y H. Takeuchi. 1995. The Knowledge-Creating Company: How Japanese Companies Create the Dynamics of Innovation. Oxford: Oxford University Press.

Oliveira, M. 2011. Ciência da Informação e Biblioteconomia: novos conteúdos e espaços de atuação. Belo Horizonte: Ed. UFMG.

Rautenberg, S., Steil, A. V. y Todesco, J. L. 2011. "Modelo de conhecimento para mapeamento de instrumentos de gestão do conhecimento e de agentes computacionais da engenharia do conhecimento". Perspectivas em Ciência da Informação 16 (3): 26-46.

Ribas, R. T. 2007. "Modelagem de processos, definição de requisitos e planejamento da implantação de um sistema de BPM em empresa têxtil.” Tesis de licenciatura, Universidade de São Paulo.

Rodrigues, L. S. 2017. Definição de requisitos. Matosinhos: Instituto Superior de Contabilidade e Administração do Porto. Slides.

Silva, S. M., F. Farinelli y M. B. Almeida. 2014. "Um roteiro para modelagem conceitual de sistemas de informação baseada em princípios ontológicos”. Trabajo presentado en el $15^{\circ}$ Encontro Nacional de Pesquisa em Ciência da Informação, Belo Horizonte, 27 a 31 de outubro.

Soares, F. M. y S. Machado. 2017. Sistema de registro de teses e dissertações do ISCAP. Matosinhos: Instituto Superior de Contabilidade e Administração do Porto. Relatório (disciplina Modelação de Sistemas da Informação, licenciatura em Ciências e Tecnologias da Documentação e Informação).

Sommerville, I. 2011. Engenharia de software. Tradução de Kalinka Oliveira e Ivan Bosnic. São Paulo: Pearson.

Villela, C. S. S. 2000. "Mapeamento de Processos como Ferramenta Reestruturação e Aprendizagem Organizacional”. Tesis de Master, Universidade Federal de Santa Catarina.

Para citar este texto:

Hamanaka, Raíssa Yuri y Filipi Miranda Soares. 2019. "A relação entre o mapeamento de processos e a modelização no contexto da gestão do conhecimento: estudo de caso aplicado em uma biblioteca digital". Investigación Bibliotecológica: archivonomía, bibliotecología e información 33 (81): 223-240. http://dx.doi.org/10.22201/iibi.24488321xe.2019.81.57997 